\title{
Deposition of colloidal asphaltene in capillary flow: experiments and mesoscopic simulation
}

\author{
Edo S. Boek*, Hemant K. Ladva, John P. Crawshaw
}

Schlumberger Cambridge Research, High Cross, Madingley Road, Cambridge CB3 OEL, UK

\author{
Johan T. Padding
}

Computational Biophysics, Dept. Science and Technology, Univ. of Twente, P.O. Box 217, 7500 AE Enschede, The Netherlands

\begin{abstract}
AUTHOR EMAIL ADDRESS: $\underline{\text { e.boek@imperial.ac.uk }}$
RECEIVED DATE

TITLE RUNNING HEAD: Deposition of colloidal asphaltene in capillary flow.
\end{abstract}

CORRESPONDING AUTHOR FOOTNOTE: e.boek@imperial.ac.uk

\begin{abstract}
The aggregation and deposition of colloidal asphaltene in reservoir rock is a significant problem in the oil industry. To obtain a fundamental understanding of this phenomenon, we have studied the deposition and aggregation of colloidal asphaltene in capillary flow by experiment and simulation. For the simulation, we have used the Stochastic Rotation Dynamics (SRD) method, in which the solvent hydrodynamics emerges from the collisions between the solvent particles, while the Brownian motion
\end{abstract}


emerges naturally from the interactions between the colloidal asphaltene particles and the solvent. The asphaltene colloids interact through a screened Coulomb potential. We vary the well depth $\varepsilon$ and the flow rate $v$ to obtain $P e^{\text {flow }} \gg>1$ (hydrodynamic interactions dominate) and $R e<<1$ (Stokes flow). In the simulations, we impose a pressure drop over the capillary length, and measure the corresponding solvent flow rate. We observe that the transient solvent flow rate decreases when the asphaltene particles become more "sticky". For a well depth $\varepsilon=2 k_{B} T$, a monolayer deposits of on the capillary wall. With increasing well depth, the capillary becomes totally blocked. The clogging is transient for $\varepsilon=5 k_{B} T$, but appears to be permanent for $\varepsilon=10-20 k_{B} T$. We compare our simulation results with flow experiments in glass capillaries, where we use extracted asphaltenes in toluene, reprecipitated with $n$-heptane. In the experiments, the dynamics of asphaltene precipitation and deposition were monitored in a slot capillary using optical microscopy under flow conditions similar to those used in the simulation. Maintaining a constant flow rate of $5 \mu \mathrm{L} \mathrm{min}{ }^{-1}$, we found that the pressure drop across the capillary first increased slowly, followed by a sharp increase, corresponding to a complete local blockage of the capillary. Doubling the flow rate to $10 \mu \mathrm{L} \mathrm{min}{ }^{-1}$, we observe that the initial deposition occurs faster, but the deposits are subsequently entrained by the flow. We calculate the change in the dimensionless permeability as a function of time for both experiment and simulation. By matching the experimental and simulation results, we obtain information about 1) the interaction potential well depth for the particular asphaltenes used in the experiments, and 2) the flow conditions associated with the asphaltene deposition process.

KEYWORDS Deposition, aggregation, asphaltenes, capillary flow, simulation. 


\section{Introduction}

The deposition of asphaltenes may cause problems in flowlines, production facilities and oil reservoirs near wellbores. It is the latter issue that we will be dealing with in this paper. Recently, a number of capillary flow experiments have been carried out to investigate asphaltene deposition. Broseta et $a .^{I}$ calculate an effective hydrodynamic thickness of a deposited asphaltene layer in flow experiments in a metal capillary, assuming a uniform thickness of the layer deposited. Wang et al. ${ }^{2}$ study the deposition of asphaltene on metallic surfaces using the homogeneous deposition hypothesis. There are several papers in the literature related to the modeling of asphaltene deposition in flowing systems. These include flow and deposition of asphaltene in production pipe lines ${ }^{3,4}$ and formation damage due to deposition in the reservoir. The latter include network models ${ }^{5}$ and Darcy scale Deep Bed Filtration continuum models. ${ }^{6}$ However, these are all phenomenological models, the parameters of which are difficult to relate to the physics of the underlying deposition process.

In this paper, we develop a predictive model of asphaltene deposition under flowing conditions, based on colloid dynamics simulations which are compared to microscopic experiments. We study asphaltene deposition in a glass capillary, which allows for direct visual observation of the deposition as a function of distance from the capillary entrance, as well as the measurement of pressure drop as a function of time. We compare our experimental results with computer simulations using a novel modelling method called Stochastic Rotation Dynamics (SRD). Experiment and simulation are compared directly by calculating the dimensionless conductivity of the capillary. The aim of our work is to contribute to a physically-based model based on colloidal interaction potentials to predict permeability damage in reservoir rock due to asphaltene deposition. 


\section{Experimental Methods / Materials and Methods}

The experimental setup comprised of a rectangular glass capillary, a microscope, constant flowrate pumps and a data acquisition system.

A rectangular open-ended borosilicate glass microcapillary with precision optical path length was used in the experiments to visualise asphaltene deposition and transport. The capillary was $100 \mathrm{~mm}$ long, 600 $\mu \mathrm{m}$ wide and $150 \mu \mathrm{m}$ deep (VD/5005-100 obtained from Camlab Ltd). The capillary wall thickness was $50 \mu \mathrm{m}$ giving a slot dimension of $500 \mu \mathrm{m}$ by $50 \mu \mathrm{m}$ and the capillary volume of $2.5 \mu \mathrm{L}$. Figure 1 shows a cross section of the capillary with near perfect right angle corners of the rectangular slot and a smooth wall finish.

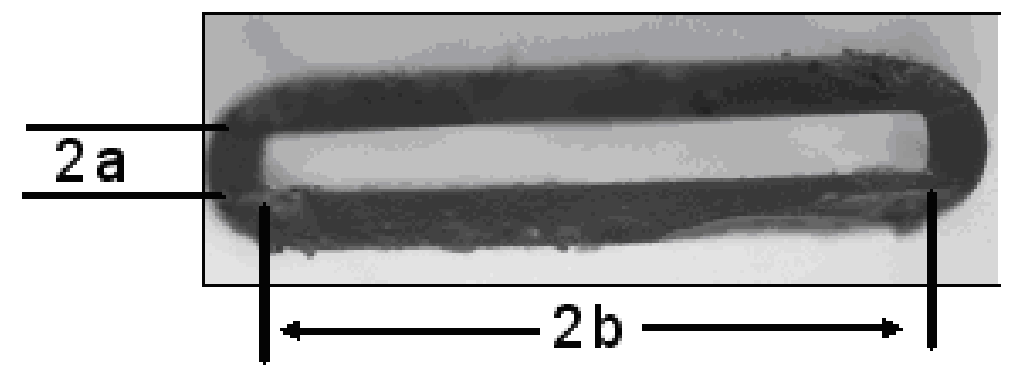

Figure 1. Cross section of a rectangular glass microcapillary.

The average shear rate was calculated using

$$
\gamma_{a v}=\frac{3 Q}{8 a^{2} b}
$$


where $Q$ is the flow rate, $a$ is half the slot depth and $b$ is half the slot width. The calculated average shear rate was $200 \mathrm{~s}^{-1}$ for the $5 \mu \mathrm{L} \mathrm{min}{ }^{-1}$ flow rate used in the experiment. At $Q=5 \mu \mathrm{L} \mathrm{min}{ }^{-1}$, the calculated residence time in the capillary was 30 seconds. Because the residence time is very short compared with the relaxation time of asphaltene molecular structure as observed in NMR experiments, we assume that the asphaltene structure does not change over the course of the experiment.

The slot capillary was inserted into the exit end of a PEEK Y-connector (P-512 from Upchurch Scientific) that was predrilled with $0.65 \mathrm{~mm}$ diameter and $2 \mathrm{~mm}$ deep hole. The hole depth was selected such that the co-injected fluids enter the capillary immediately after the mixing point. The residence time was $<0.5$ second from the point of mixing to the capillary entrance. The flow channel diameter of the Y connector was $0.5 \mathrm{~mm}$. The capillary was sealed using a silicon sealant (555-588 from RS) and left to set for 24 hours before using the assembly for the experiment. The Y connector was subsequently secured onto an in-house built microscope glass slide holder designed for light transmission. The capillary was cemented with its wide side lying flat onto the glass slide using quick set epoxy. The whole assembly was mounted onto the microscope stage and the two co-injection fluid inlet lines were connected to the $\mathrm{Y}$ connector ensuring that there was no trapped air. The fluids were injected at constant flow rate into the capillary via the Y connector using micro-syringe pumps (BS8000 from Braintree Scientific). The pressure drop across the capillary was monitored using a 1 bar pressure gauge (3488065 from RS) and the data recorded to a computer.

A Zeiss microscope (AxioImager Z1 AX10) for imaging in transmitted light mode was used in the experiments. The microscope equipped with 10x (A Plan 10x/0.25 Ph1) and 100x (EC Plan-Neofluar 100x/1.30 oil M27) objectives allowed imaging using brightfield, phase contrast and depth imaging contrast (DIC). A monochrome camera (QICAM-QIC-F-CLR-12-C), a motorized stage (Prior H101A) and Image-Pro Plus imaging software from Media Cybernetics was used for qualitative and quantitative image analysis. 


\section{Experimental method}

In our experiments, we used asphaltenes extracted from a Cold Lake crude oil sample. The Cold Lake crude oil, as received from the field, contained $24.3 \mathrm{wt} \%$ water, determined using Karl Fischer titration. The high percentage of water present in the crude may be an artefact due to the use of steam for production of the oil. Figure 2 shows the water present in the neat crude oil. The Cold Lake crude oil was first dewatered before use and its composition measured in duplicate as shown in Table 1. A mass balance gave an error of $4 \mathrm{wt} \%$ for both samples.

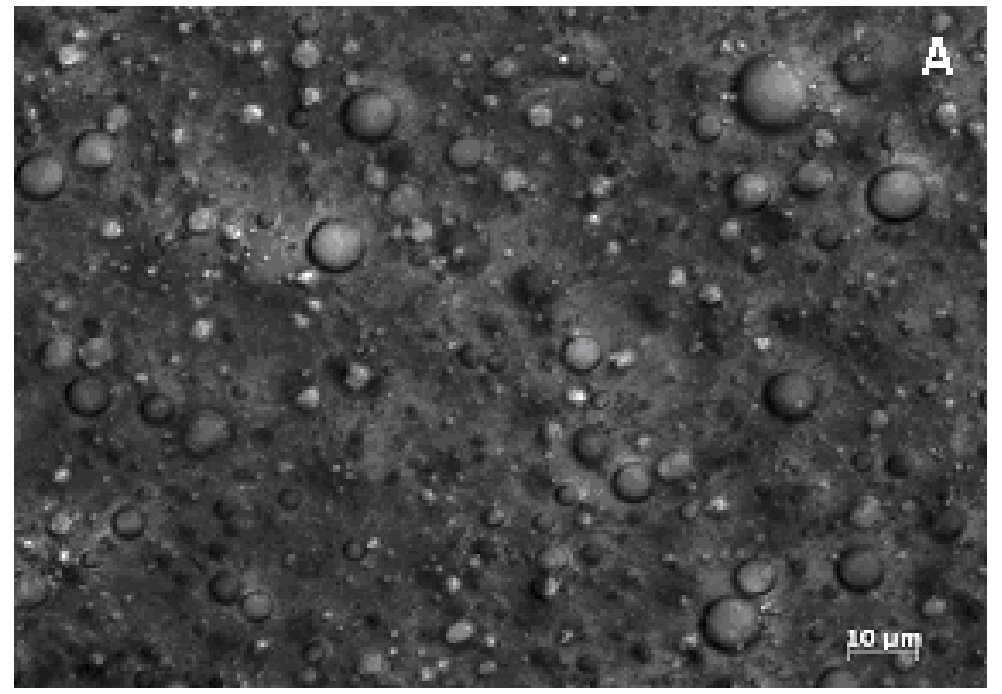

Figure 2. Water droplets in neat Cold Lake crude oil

$\begin{array}{lll} & \text { Sample 1 wt } \% & \text { Sample 2 wt } \% \\ \text { Maltenes } & 84.67 & 84.69 \\ \text { Asphaltenes } & 11.12 & 11.14 \\ \text { Inorganics } & 0.06 & 0.08 \\ \text { Volatiles } & 0.45 & 0.45 \\ \text { Total wt\% } & 96.30 & 96.36\end{array}$

Table 1. Composition of Cold Lake crude oil measured in duplicate 


\section{Fluid preparation}

First, the asphaltene was extracted from the crude oil using $n$-heptane in a ratio of $40 \mathrm{ml}$ heptane to 1 $g$ of dewatered oil. The model fluid system used in the deposition experiments comprised of the extracted asphaltenes dissolved in toluene (Aldrich). The experiments were conducted with a dilute system at $1 \mathrm{gL}^{-1}$ asphaltene concentration. The extracted asphaltenes were dissolved in toluene at $1 \mathrm{gL}^{-1}$ concentration and sonicated for 30 minutes at room temperature to ensure that all the asphaltenes were dissolved. The solution was tested in the microscope using a 10x objective and no measurable particles were detected; i.e. no asphaltene particles greater than 0.5 micron were present in toluene samples. The model fluid system was prepared immediately prior to conducting the experiments. The solvent used to precipitate the asphaltenes from the model fluid system was $n$-heptane chromasolv (Aldrich) of $>99 \%$ purity.

\section{Experiments}

We have investigated the effect of

- Constant flow rate at $5 \mu \mathrm{L} \min ^{-1}$ of n-heptane and $1 \mathrm{~g} \mathrm{~L}^{-1}$ concentration of asphaltene in toluene at 60:40 ratio (in triplicate).

- Doubling the flowrate to $10 \mu \mathrm{L} \mathrm{min}{ }^{-1}$ of $\mathrm{n}$-heptane and $1 \mathrm{~g} \mathrm{~L}^{-1}$ concentration of asphaltene in toluene at $60: 40$ ratio.

The co-injected fluids are toluene with dissolved asphaltene from one end of the $Y$-connector and pure heptane from the other end. Baseline pressure drop measurements were made at $25^{\circ} \mathrm{C}$ for different flow rates of a 60:40 mixture of $n$-heptane and toluene. The capillary was not pretreated in any way and used as received from the suppliers. 


\section{Constant flow rate of $5 \mu \mathrm{L} \mathrm{m^{-1 }}$ of n-heptane and asphaltene in toluene}

Three repeat experiments (A, B and C) were carried out at room temperature with co injection of asphaltenes in toluene $\left(1 \mathrm{gL}^{-1}\right.$ concentration) at $2 \mu \mathrm{L} \cdot \mathrm{min}^{-1}$ flow rate and $n$-heptane at $3 \mu \mathrm{L} \cdot \mathrm{min}^{-1}$ flow rate. Figure 3 shows the pressure history for the three repeat experiments.

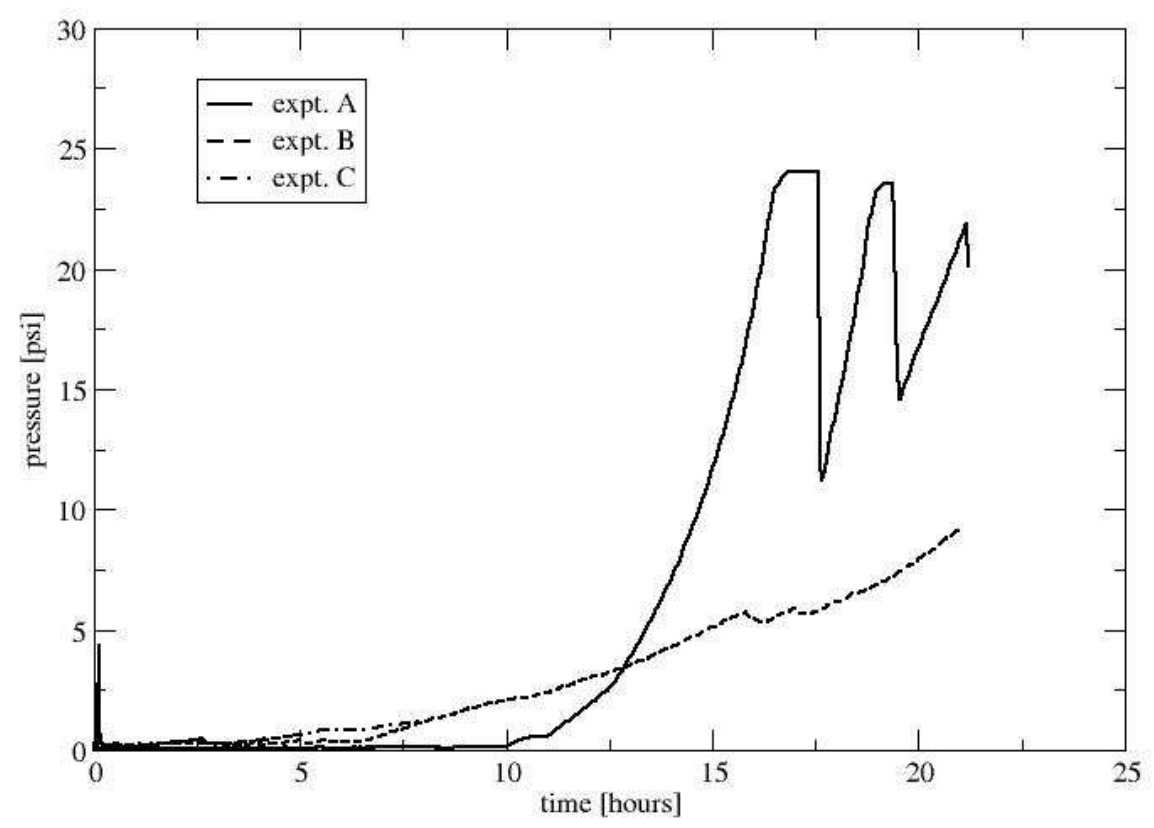

Figure 3. Pressure drop history for constant flow rate of $5 \mu \mathrm{L} \mathrm{min}{ }^{-1}$ for 3 repeat experiments.

For all three repeat experiments, the early time behaviour shows a slow increase in the pressure drop. We hypothesize that this is due to the continuous deposition of asphaltenes. For later times, the slope of these curves, representing the rate of asphaltene deposition, is different for the three experiments. In particular for experiment A, there seems to an initial period of slow deposition (induction time) followed by a regime where deposition rates increase strongly. These observations are reminiscent of a nucleation process. 
Our hypothesis regarding the rapid retention of asphaltenes and subsequent erosion from the pressure data was confirmed by the microscope images captured $3 \mathrm{~cm}$ from the capillary inlet at regular time interval. Figure 4 shows the amount of asphaltene retention as a function of with time for experiment A. In this figure, we observe for early times that asphaltene is depositing uniformly onto the capillary walls. Subsequently, large flocs were formed (approximately $100 \mu \mathrm{m}$ in size) and transported downstream. The mobile large flocs eroded the deposited asphaltenes from the wall to the extent where clear channels were visible, as shown in Figure 4 at 290 minutes. We note here already that very similar behaviour is observed in the simulations which will be described in the following section.

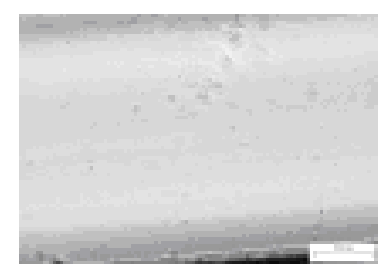

$0 \mathrm{~min}$

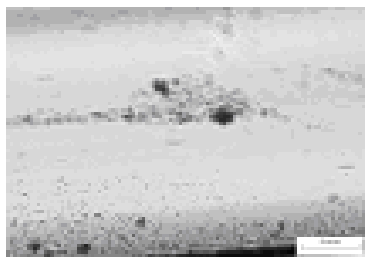

$260 \mathrm{~min}$

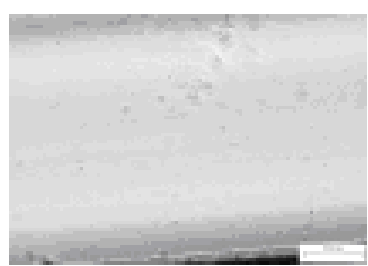

$5 \min$

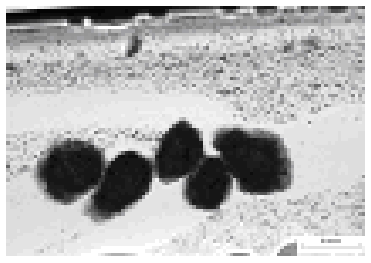

$290 \mathrm{~min}$

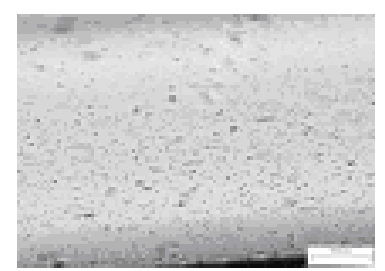

$90 \mathrm{~min}$

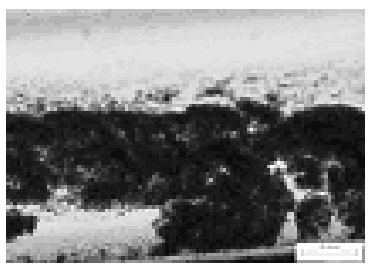

$490 \mathrm{~min}$

Figure 4. Asphaltene retention as a function of time for Experiment A.

A maximum pressure of 24 psi was reached in experiment A, followed by a series of pressure cycles. This behavior corresponds to increased asphaltene retention and asphaltene deposition-erosion / entrainment cycles. The pressure cycles however caused only partial removal of the deposited asphaltenes. 


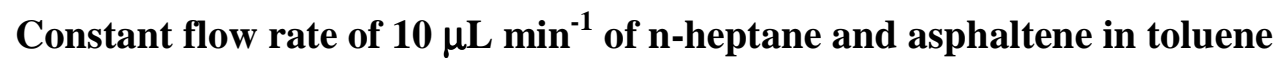

This experiment was carried out to study the effect of flow rate on deposition. We kept the flow rate of $n$-heptane at $6 \mu \mathrm{L} \min ^{-1}$ and asphaltene in toluene $\left(1 \mathrm{~g} \mathrm{~L}^{-1}\right)$ at $4 \mu \mathrm{L} \mathrm{min}{ }^{-1}$ constant throughout the experiment. Figure 5 shows the pressure history. The pressure increases at the rate of $1.15 \mathrm{psi} \mathrm{hr}^{-1}$ for the first 6 hours of the experiment and subsequently after 8.5 hours the rate increases sharply to 12.25 $\mathrm{psi} \mathrm{hr}^{-1}$. These rates are higher than the ones observed in the previous experiment for a lower flow rate of $5 \mu \mathrm{L} \mathrm{min}{ }^{-1}$. This suggests that the aggregation / deposition rates increase with increasing flow rate. We note that this is in qualitative agreement with recent experimental work on asphaltene aggregation in shear flow: Rahmani et al. ${ }^{7}$ observed that, for early times, the largest aggregates are observed for the highest imposed shear rate. The authors hypothesize that, at higher shear rates, the asphaltenes have a higher collision frequency and therefore an increased likelihood to aggregate.

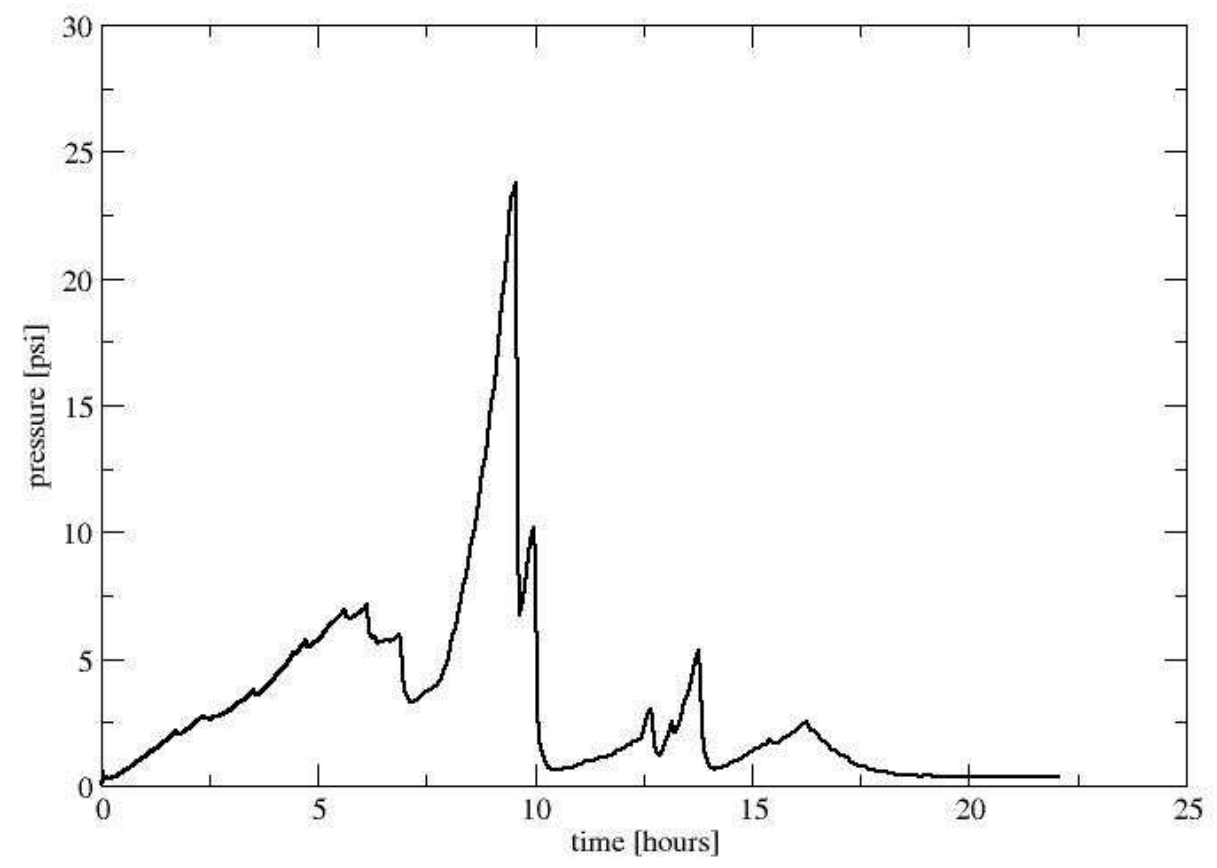

Figure 5. Pressure profile for the higher flowrate of $10 \mu \mathrm{L} \mathrm{min}{ }^{-1}$ 
Also, we observe directly from light microscopy that the amount of asphaltene deposited decreases with distance form the capillary inlet. This is shown in Figure 6. Note that Broseta et al. ${ }^{\text {Error! Bookmark not }}$ defined. calculate an effective hydrodynamic thickness of a deposited asphaltene layer in their capillary flow experiments, assuming a uniform thickness of the layer deposited. Our experiments indicate that such an assumption may not be justified.

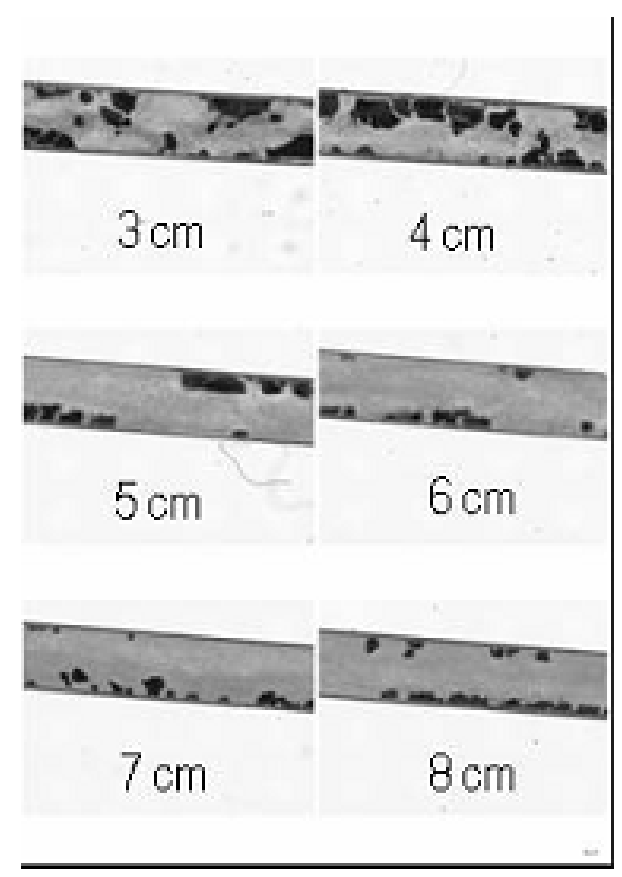

Figure 6. Deposition decreases as a function of distance from the capillary entracnce at the end of the experiment - flow rate $10 \mu \mathrm{L} \mathrm{min}^{-1}$

The pressure buildup for the first 9.5 hours followed by the sharp decrease in pressure is reflected by the deposition and subsequent erosion as shown by the sequence of images collected every hour $3 \mathrm{~cm}$ from the capillary entrance. The image taken at 10 hours clearly shows significant decrease in the deposited asphaltenes. This is shown in Figure 7. 


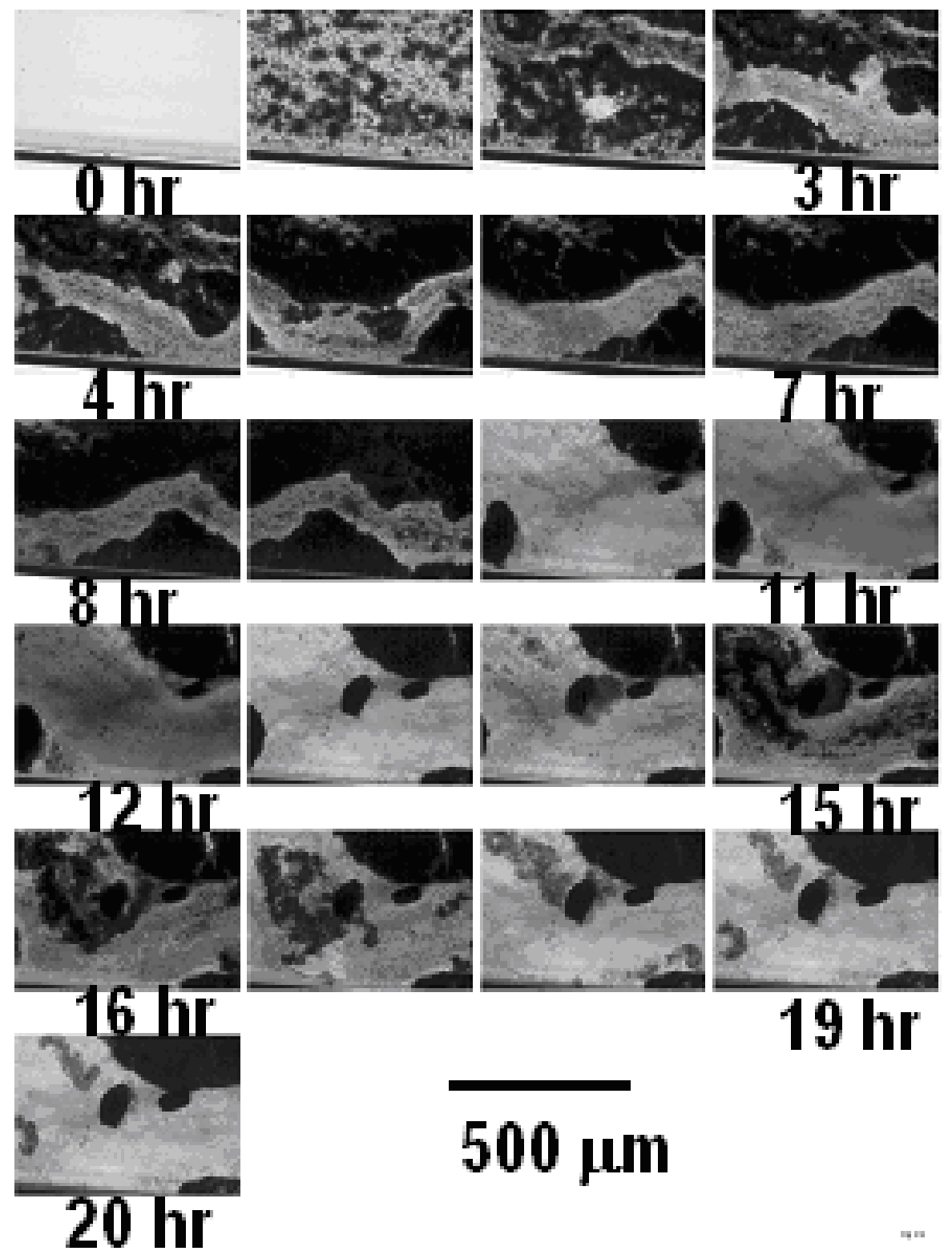

Figure 7. Images taken every hour at $3 \mathrm{~cm}$ from the capillary inlet - flow rate $10 \mu \mathrm{L} \mathrm{min}{ }^{-1}$ 


\section{Simulation Methods}

We will first describe the simulation technique used, followed by the definition of the colloidal interaction potentials.

\section{- Stochastic Rotation Dynamics (SRD)}

We have used a particulate simulation technique called Stochastic Rotation Dynamics (SRD). This method has recently been developed to solve the hydrodynamics of complex fluids. ${ }^{8}$ Although SRD is a fairly recent method, its statistical mechanical and hydrodynamic fundamentals have been thoroughly studied and validated. ${ }^{9,10}$ It is a mesoscopic particulate method where the solvent is represented by $N_{f}$ point-like (ideal gas) particles. It is similar in spirit to the lattice-Boltzmann (LB) method. ${ }^{11,12}$ In contrast to LB however, it includes Brownian motion which emerges naturally from the thermal fluctuations of the SRD particles $\left(N_{f}\right)$. This makes the SRD method particularly useful to study the hydrodynamics of colloids such as oilfield asphaltene and wax particles. The colloidal particles are described by means of a coarse-grained Molecular Dynamics method ${ }^{10}$ which will be described in the following section. The SRD hydrodynamics emerges from collisions between solvent particles in coarse-grained cells at coarse-grained time intervals. It is a simple and computationally cheap algorithm $\left(O\left(N_{f}\right)\right)$, which can be easily coupled to solutes such as polymers and colloids. The algorithm proceeds in two steps. ${ }^{13}$ In the first of these, a free streaming step, the positions of the solvent particles, $\boldsymbol{r}_{i}(t)$, are updated simultaneously according to

$$
\mathbf{r}_{i}(t+\delta t)=\mathbf{r}_{i}(t)+\mathbf{v}_{i}(t) \delta t
$$

where $v_{i}(t)$ is the velocity of a particle and $\delta t$ is the discretised time step of the solvent. 
The second part of the algorithm is the collision step. The simulation system is coarse-grained into cells. Stochastic multiparticle collisions are performed within each individual cell, by rotating the velocity of each particle relative to the center-of-mass velocity $\boldsymbol{v}_{c m}(t)$ of all the particles within that cell:

$$
\mathbf{v}_{i}(t+\delta t)=\mathbf{v}_{c m}(t)+\mathbf{R}\left(\mathbf{v}_{i}(t)-\mathbf{v}_{c m}(t)\right)
$$

$\mathbf{R}$ is a rotation matrix which rotates velocities by a fixed angle $\alpha$ around an axis generated randomly for each cell and at each time step. The aim of the collision step is to transfer momentum between the particles while conserving the total momentum and energy of each cell. Because mass, momentum and energy are conserved locally, the thermohydrodynamic equations of motions are captured in the continuum limit. ${ }^{8}$ Hence hydrodynamic interactions can be propagated by the solvent. Note, however, that any molecular details of the solvent are excluded - this allows the hydrodynamic interactions to be modelled with minimal computational expense. This procedure conserves mass, momentum and energy and yields the correct hydrodynamic behaviour, in the sense that the Navier-Stokes equations are recovered. ${ }^{14}$ The fluid particles only interact with each other through the rotation procedure, which can be viewed as a coarse-graining of particle collisions over space and time. For this reason, the SRD solvent should not be considered as molecules, but rather as a Navier-Stokes solver that naturally includes Brownian noise. The SRD simulation technique has been applied to colloids in solution, ${ }^{8}$ colloidal sedimentation, ${ }^{14}$ clay particles, polymer fluids, amphiphilic systems, flow in porous media, binary fluid mixtures, flow around solid objects and reactive fluids.

Here, we consider the aggregation and deposition of asphaltene colloidal particles in capillary flow in 2 dimensions (2-D) using the SRD method. The solvent is represented by SRD point particles as described above. Asphaltene particles are defined in the fluid as colloidal discs (in 2-D) using the Molecular Dynamics (MD) algorithm. ${ }^{10}$ We fix the length scale of the model by choosing the radius $R$ of the asphaltene colloids. 


\section{- colloidal interactions}

It is not straightforward to obtain asphaltene colloid-colloid (c-c) and colloid-wall (c-w) interactions. For this reason, we choose a model interaction potential with variable well depth $\varepsilon_{\mathrm{cc}}$, corresponding with a screened Coulomb potential, according to the Yukawa functional form:

$$
\phi_{c c}(r)=\varepsilon_{1} \frac{\sigma_{1}}{r} \exp \left(\kappa_{1}\left(r-\sigma_{1}\right)\right)+\varepsilon_{2} \frac{\sigma_{2}}{r} \exp \left(\kappa_{2}\left(r-\sigma_{2}\right)\right)
$$

In this way, the colloid-colloid interactions are defined in a classical van der Waals manner ${ }^{15}$ through a combination of attractive and repulsive interactions. Here $\varepsilon_{1}$ and $\varepsilon_{2}$ are the parameters for attraction and repulsion, respectively, $r$ is the distance between the colloids, $\kappa^{-1}$ is the Debye screening length and $\sigma$ is the colloid diameter. A typical way to parameterize this equation is shown in Figure 8, where the depth of the potential well at the minimum, $\varepsilon_{\mathrm{cc}}$, is varied between $2,5,10,20$ and $50 \mathrm{k}_{\mathrm{B}} \mathrm{T}$, by choosing appropriate values of $\varepsilon_{1}$ and $\varepsilon_{2}$.

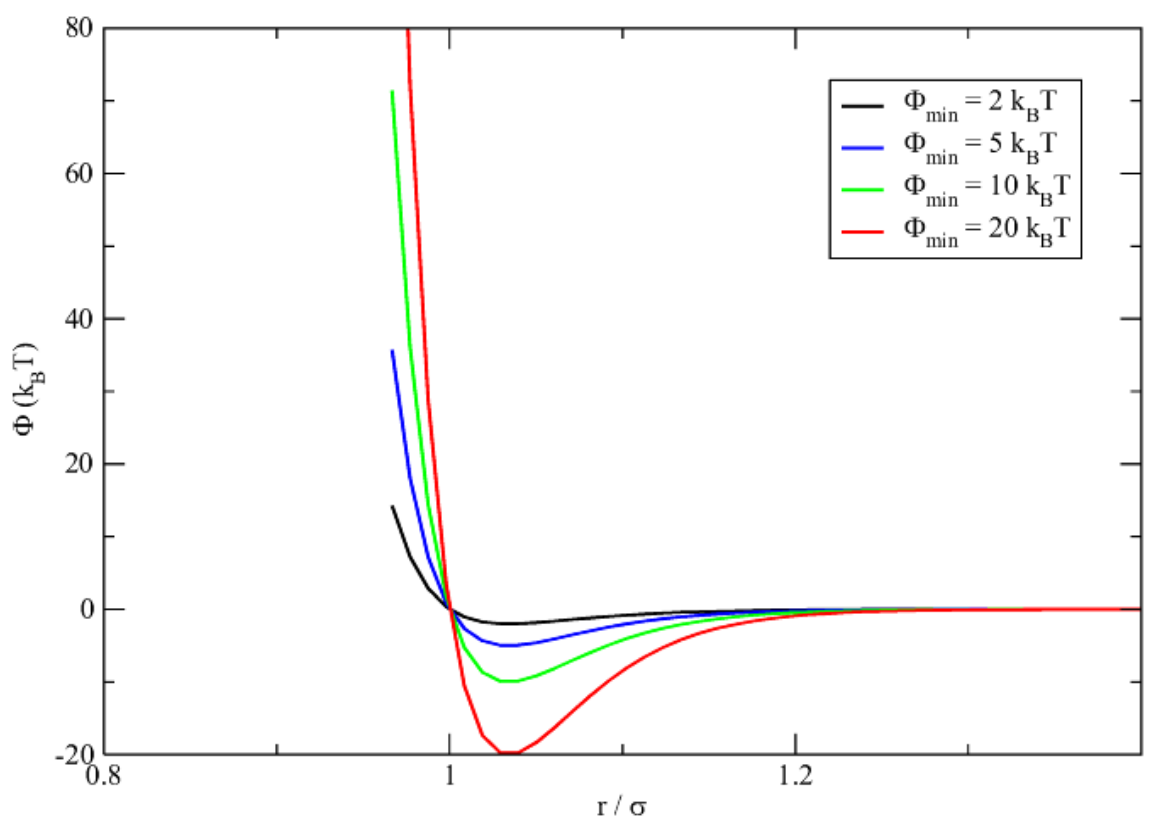

Figure 8. colloid-colloid interaction potential. By appropriately choosing the parameters for repulsion and attraction, the depth of the potential well is varied between $2,5,10$ and $20 \mathrm{k}_{\mathrm{B}} \mathrm{T}$. 
Note that it is the depth of this well that determines the degree of "stickiness" between the colloidal particles; the deeper the potential well, the stronger the shear forces required to break up the aggregate.

Note that we have used a Double Yukawa (DY) functional form for the colloid-colloid interactions. This is a functional form commonly used for the effective interaction between colloidal aggregates. ${ }^{16}$ In general, these effective interactions result from a sum over the contributions of the individual molecules, which are normally considered to have a Lennard-Jones 6-12 functional form. In particular, it can be shown that the DY functional form gives a very good fit for the sum over the van der Waals interactions between $C 60$ colloidal particles ${ }^{17}$. We assume here that also the effective interactions between colloidal asphaltene aggregates can be described by a DY potential. In addition to the van der Waals interactions, we have a Coulomb interaction, which is screened by the oil phase. It can be shown that, for an oil phase with a dielectric constant $\varepsilon_{r}=3$, the electrostatic interactions will decay to order $k_{B} T$ over a distance of $19 \mathrm{~nm}$. This shows that the range of the electrostatic interactions is much smaller than the size of our asphaltene aggregate particles with a diameter of $3000 \mathrm{~nm}$.

We note that the DY form of the colloid-colloid interaction may not be exactly the same as the real effective interactions between 3 micrometer sized clusters of asphaltene. ${ }^{20}$ However, since the range of attraction is extremely small compared to the size of the colloid, the precise form of the interaction does not matter. It can be shown that only the integral over the attractive well, i.e. the second virial coefficient, is of influence on the thermodynamic and aggregation properties. ${ }^{16}$ Because a very small range is computationally too expensive (extremely small time steps in Molecular Dynamics) we choose a slightly larger range of attraction, but still small compared to the particle diameter. 
Obtaining the appropriate value for the effective potential well depth to represent the asphaltenes requires some consideration. In this paper, our main aim is to obtain a correct description of the balance between the deposition of asphaltene aggregates and the stress induced by the fluid flow. In an ideal world, this should be done using a full molecular description of the asphaltenes, with atomistic resolution. ${ }^{18}$ Unfortunately, it is very difficult to achieve this goal using Molecular Dynamics (MD) simulations, due to computational limitations of current computers. In order to make progress in a timely fashion, we have used a coarse-grained description of the asphaltene aggregates, using an effective interaction potential with an attractive well, the depth of which can be tuned. In this paper, we adopt the pragmatic approach of estimating the value of the potential well depth by comparing the results of our capillary experiments to the simulations. We will report on MD simulations of asphaltenes with molecular resolution in a separate paper. ${ }^{19}$

For the colloid-wall (c-w) interactions, we consider two different scenarios. First, we consider an infinitely deep potential well $\varepsilon_{\mathrm{cw}}$, which means that the particles will stick irreversibly to the capillary wall. This corresponds with adsorption on a metal surface. Second, we assume that the capillary wall is covered by a thin layer of asphaltene particles, so that the c-w interaction is identical to the c-c interaction. This scenario corresponds with adsorption on a mineral (rock) surface or a glass capillary. 


\section{- mechanism of asphaltene aggregation}

Yudin et al. ${ }^{20}$ studied the mechanisms of asphaltene aggregation in toluene-heptane mixtures using dynamic light scattering. They observed that, for asphaltene concentrations above the critical aggregate concentration (CAC), two different mechanisms of aggregation occur, as a function of time. In the initial stages, where the mean radius of the aggregates is smaller than $1 \mu \mathrm{m}$, the aggregation can be described by a Reaction Limited Aggretation (RLA) process. This means that not every contact between two particles results in their sticking. In terms of colloidal interaction potentials (see above) this is usually described by means of an activation barrier between the free and aggregated states. In the later stages, where the mean radius of the aggregates is equal to or larger than $3 \mu \mathrm{m}$, the aggregation can be described by a Diffusion Limited Aggregation (DLA) process. This means that every contact between the particles results in their association. Based on microscopic and light scattering ${ }^{20}$ observations of asphaltene aggregate sizes, we consider here asphaltene particles with a radius of $3 \mu \mathrm{m}$, interacting by means of a DLA potential without activation barrier. 


\section{- dimensional analysis}

In the capillary flow experiment, we consider a volumetric flow rate $Q=5 \mu \mathrm{l} / \mathrm{min}$. Using the capillary surface area $A=(50 \times 500) \mu \mathrm{m}^{2}$, this corresponds with a linear flow rate $v_{\text {flow }}=Q / A=1 / 300$ $\mathrm{m} / \mathrm{s}$.

Assuming a colloid aggregate radius of $3 \mu \mathrm{m}$, we calculate the diffusion coefficient $D$ from the Stokes-Einstein equation

$$
D=\frac{k_{B} T}{6 \pi \eta R}=7.3 * 10^{-14} \mathrm{~m}^{2} / \mathrm{s}
$$

We now calculate the Peclet number for flow $P e^{\text {flow }}$ as

$$
P e^{\text {flow }}=\frac{v^{\text {flow }} R}{D}=\frac{v^{\text {flow }} R^{2} 6 \pi \eta}{k_{B} T}
$$

For the experiment, we find $P e^{\text {flow }}=1.4 * 10^{5}$. This means that hydrodynamic interactions are much more important than Brownian diffusion. Note that in the simulations, with the current set of parameters we can achieve Peclet numbers up to 10 . While not corresponding directly to the experiment, because $P e^{\text {flow }}>>1$ in both simulation and experiment, we expect that hydrodynamic interactions dominate in both cases and it is justified to make qualitative comparisons.

Finally, we calculate the Reynolds number for the particles $R e^{p}$ as

$$
\operatorname{Re}^{p}=\frac{v^{\text {flow }} R \rho}{\eta}=0.01
$$

Because $R e^{p}<<1$, we are in the Stokes flow limit, where inertial effects are unimportant. This regime is typical for flow in porous media. 


\section{- time scales}

We will now show that the time scale of the simulation can be matched with the time scale of the experiment, by considering the diffusion of the colloidal particles.

First, we consider the time required for an asphaltene colloid to diffuse over its own radius, $\tau_{\mathrm{D}}$ :

$$
\tau_{D}=\frac{R^{2}}{D}
$$

where $R$ is the colloid radius and $D$ is the self-diffusion coefficient. Assuming $R=3 \mu \mathrm{m}$ and using the value for $D$ obtained in the previous section, we find $\tau_{\mathrm{D}}=124 \mathrm{~s}$.

We determine the self-diffusion coefficient in 2 dimensions consistently from a simulation of a single colloid in a capillary by calculating the velocity autocorrelation function (VACF). ${ }^{21}$ From the calculation, we find $\tau_{\mathrm{D}}=75$ (in time units $\mathrm{a}_{0}(\mathrm{~m} / \mathrm{kT})^{1 / 2}$ used in the program), so that $\tau_{\mathrm{SRD}}=124 / 75=$ 1.65 s. One Molecular Dynamics (MD) time step of 0.01 SRD time units therefore corresponds to $0.0165 \mathrm{~s}$. Typically, we carry out a simulation run for 10 million MD time steps, which ensures that a steady state is achieved. This is equivalent to $10^{7} \times 0.0165 \mathrm{~s} \cong 45$ hours. This time scale is comparable with the time scale of the flow experiments. 


\section{- simulation details}

We will now describe the technical details of the simulations. We use a two-dimensional simulation box of dimensions $L_{x} \times L_{y}=75 \times 450$ SRD cells, at a number density $\rho$ of 5 SRD particles per cell. ${ }^{14}$ Periodic boundary conditions are used along the direction of the flow, $y$. This means that we are effectively simulating an infinitely long capillary. We consider a constant number of asphaltene colloidal particles. These are inserted randomly in the SRD fluid at a concentration of $4 \%$ by volume. The colloidal particles have a fixed diameter $\sigma_{\mathrm{cc}}$ of 3 SRD cells. The solvent has a viscosity $\eta=10^{-3}$ Pa.s.

The solvent-colloid radius is always slightly smaller than the colloid-colloid interaction radius (1.3 and 1.5 SRD cells, respectively). This ensures that the simulation is effectively still representative of a three-dimensional system, not in terms of blocking, but hydrodynamically. A similiar method has been used successfully in recent LB-DEM simulations ${ }^{22}$. Essentially, this means that solvent flow is still possible through the thin solvent layer. There will always remain a base permeability in this model, even in the case of complete blockage.

We generate Poiseuille flow in the capillary by imposing a body force $g_{S R D}$ on the SRD particles. In the absence of blockage, the required body force to attain a flow velocity $v^{\text {flow }}$ is given by:

$$
g_{S R D}=\frac{16 \eta v^{\text {flow }}}{\rho L_{x}^{2}}
$$

This corresponds with a constant pressure drop

$$
\frac{\Delta P}{\Delta x}=\rho g_{S R D}
$$

and the corresponding solvent velocity $v_{\text {solv }}$ is measured. Note that in the experiment, we impose a constant flow rate and measure the corresponding pressure drop. Direct comparison between the experimental and simulation results is possible by calculating the dimensionless conductivity. This will be shown in the following section. 


\section{Simulation Results}

We consider two different scenarios for the colloid-wall (c-w) interactions.

First, we present a set of simulation results with an infinitely deep potential well $\varepsilon_{\mathrm{cw}}=-\infty$, which means that the particles will stick irreversibly to the capillary wall. This corresponds with adsorption on a metal surface. In this set of simulations, we vary the colloid-colloid well depth $\varepsilon_{\mathrm{cc}}$ systematically from $2,5,10,20$ to $50 k_{B} T$.

We find that the transient solvent flow rate decreases when the asphaltene particles become more "sticky". For a well depth $\varepsilon_{\mathrm{cc}}=2 k_{B} T$, we find the deposition of a monolayer on the capillary wall. With increasing well depth, we find that the capillary becomes totally blocked. The clogging is transient for $\varepsilon_{\mathrm{cc}}=5 k_{B} T$, but appears to be permanent for $\varepsilon_{\mathrm{cc}}=10-20 k_{B} T$. Simulation snapshots taken at the end of the runs of 10 million steps each show are presented in Figure 9.
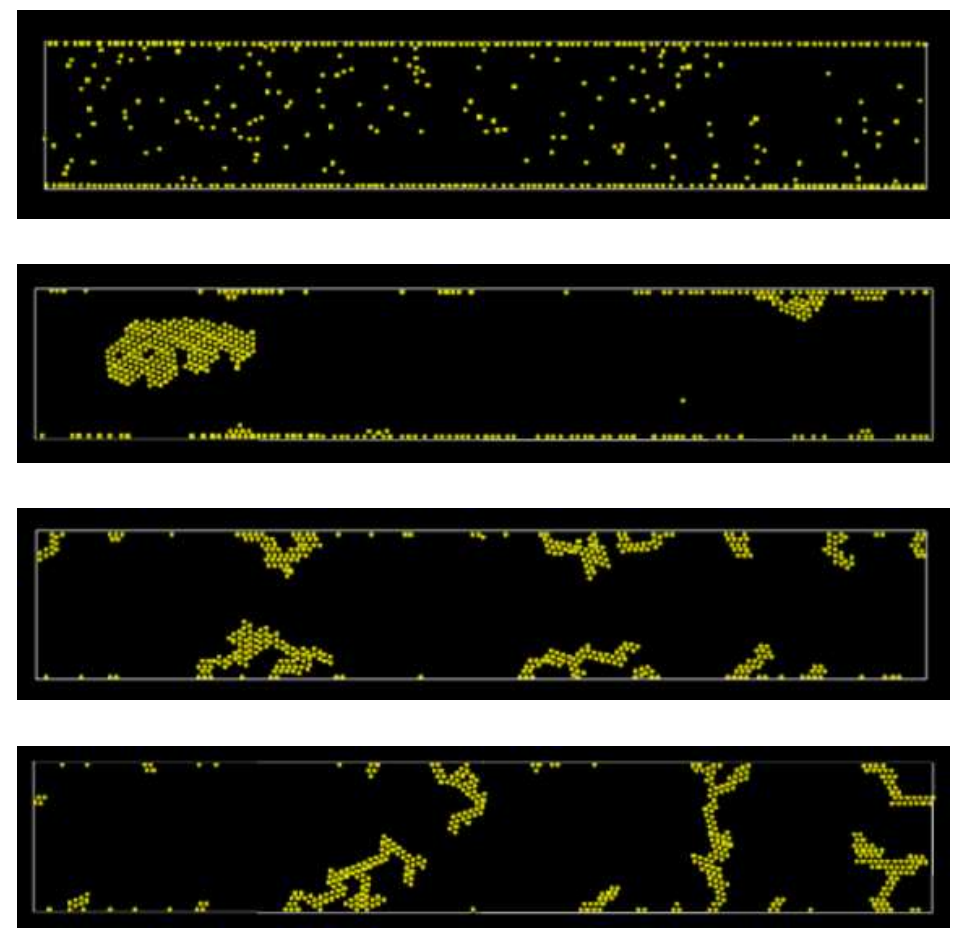

Figure 9: Simulation snapshots for steady state behaviour, after 10 million time steps, as a function of increasing c-c potential well depths: from top to bottom $\varepsilon_{\mathrm{cc}}=2,5,10$ and $20 k_{B} T$. 
In Figure 10, we present the numerically measured solvent velocity as a function of time, for $\varepsilon_{\mathrm{cc}}=2$, 5, 10 and $20 k_{B} T$ respectively. It can be clearly observed that the final fluid velocity decreases with increasing potential well depth $\varepsilon_{\mathrm{cc}}$.
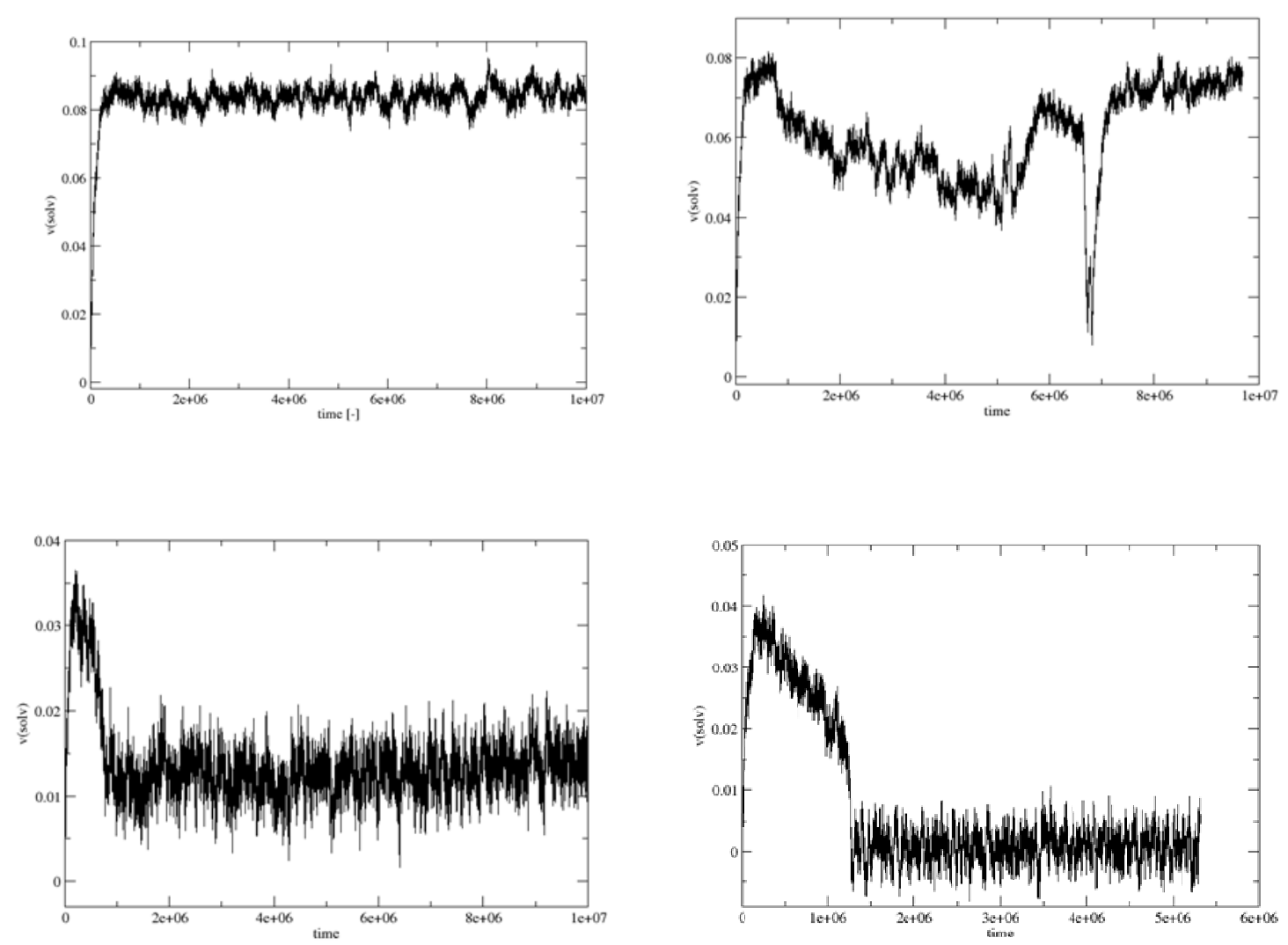

Figure 10. Solvent velocity as a function of time for $\varepsilon_{\mathrm{cc}}=2 k_{B} T$ (top left), $5 k_{B} T$ (top right), $10 k_{B} T$ (bottom left) and $20 k_{B} T$ (bottom right) respectively.

Subsequently, we present results for the pressure drop as a function of flow rate, as measured from steady state simulation conditions for the smooth wall in Figure 11. 


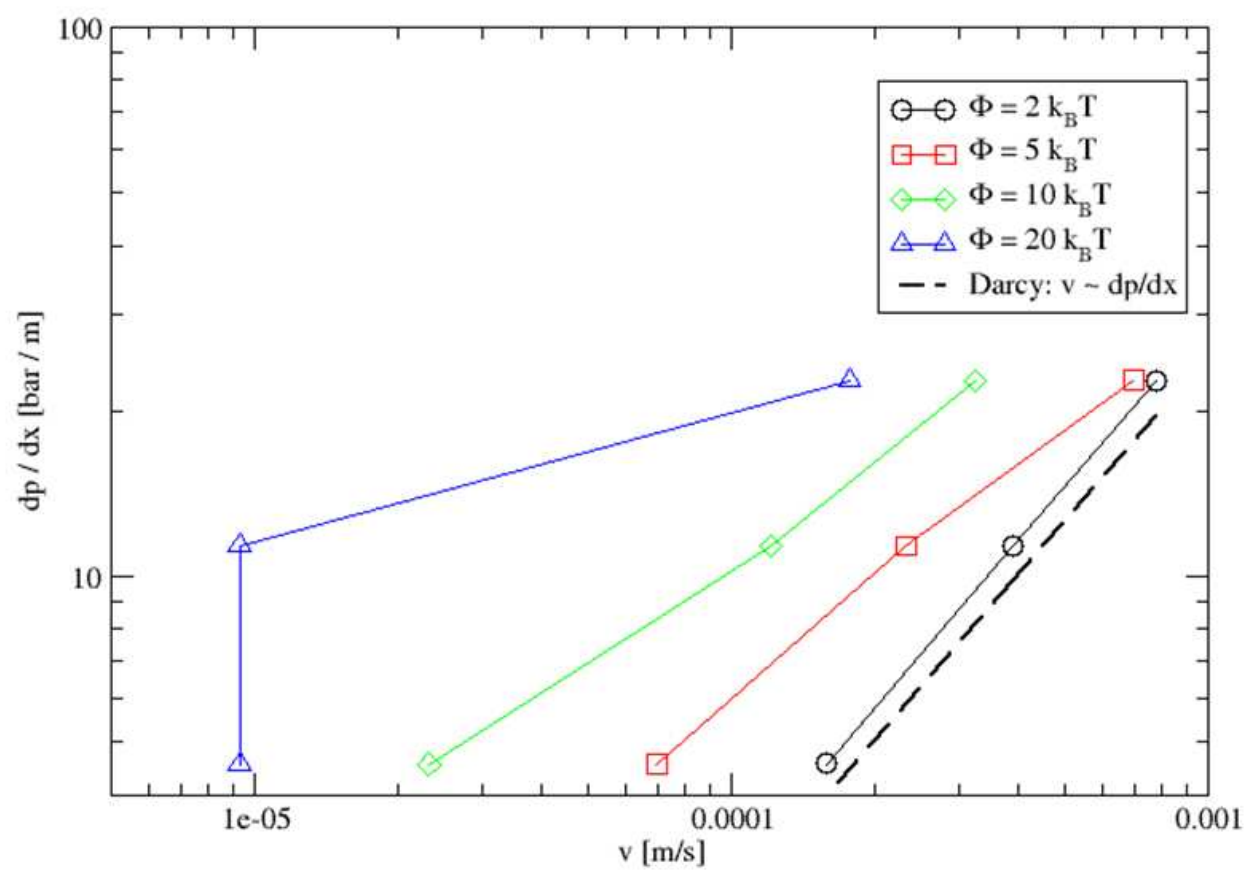

Figure 11: Pressure drop as a function of flow rate in a capilary with an infinitely deep interaction potential well $\varepsilon_{\mathrm{cw}}=-\infty$.

First, we observe that for $\varepsilon_{\mathrm{cc}}=2 k_{B} T$, the slope on a $\log$-log scale is approximately 1, indicating Darcy flow behaviour. This is in accordance with expectations. For deeper interaction potential well depths, however, we expect the graph of the pressure drop against flow rate to deviate from linear behaviour. In detail, we expect to observe a higher pressure drop at the same flow rate, due to local deposition. In Figure 11, we indeed observe this behaviour for $\varepsilon_{\mathrm{cc}}=5$ and $\varepsilon_{\mathrm{cc}}=10 k_{B} T$. With deepening of the interaction potential well, the deviation from Darcy behaviour (slope 1 on the log-log scale) is found to become larger and larger. For the deepest interaction potential well $\left(\varepsilon_{\mathrm{cc}}=20 k_{B} T\right)$, the pressure drop quickly changes at the lowest flow rates. This is because the capillary is fully blocked for the two lowest values of the pressure drop imposed. Here we observe the base permeability of the capillary, for the case of complete blockage.

In a second set of simulations, we assume that the capillary wall is now a mineral (rock) surface or a glass capillary, as in the experiments described in the previous section. In practice, this means that the 
interaction between the asphaltene particles and the capillary walls is much weaker than in the previous case. In the simulation, we model the glass capillary by assuming that the wall is covered by a thin layer of asphaltene particles, so that the $\mathrm{c}-\mathrm{w}$ interaction is identical to the c-c interaction: $\varepsilon_{\mathrm{cw}}=\varepsilon_{\mathrm{cc}}$. We first make a qualitative comparison between the flow experiments and the simulations by inspection of the time frames. The experiment shows big asphaltene aggregates with a diameter of 10-100 $\mu \mathrm{m}$ flowing down the capillary, forming transient blockages. This can be best compared with a set of simulations using $\varepsilon_{\mathrm{cw}}=\varepsilon_{\mathrm{cc}}=5 k_{B} T$ and $P e=10$, where the same phenomenology can be observed from the simulation trajectories.

To make a more quantitative comparison, we will make use of the time scale analysis presented in the previous section. We transform both the experimental time series for the pressure drop $d p / d x$ and the simulation time series for the measured velocity $v$ to dimensionless conductivity $k / k_{0}$ using Darcy's law. First, we consider the experimental results for a volumetric flow rate $Q=5 \mu \mathrm{l} / \mathrm{min}$. The experimental values of the dimensionless conductivity are presented in Figure 12, together with the corresponding simulation results for the case $\varepsilon_{\mathrm{cw}}=\varepsilon_{\mathrm{cc}}=10 k_{B} T$ and $P e=5$. 


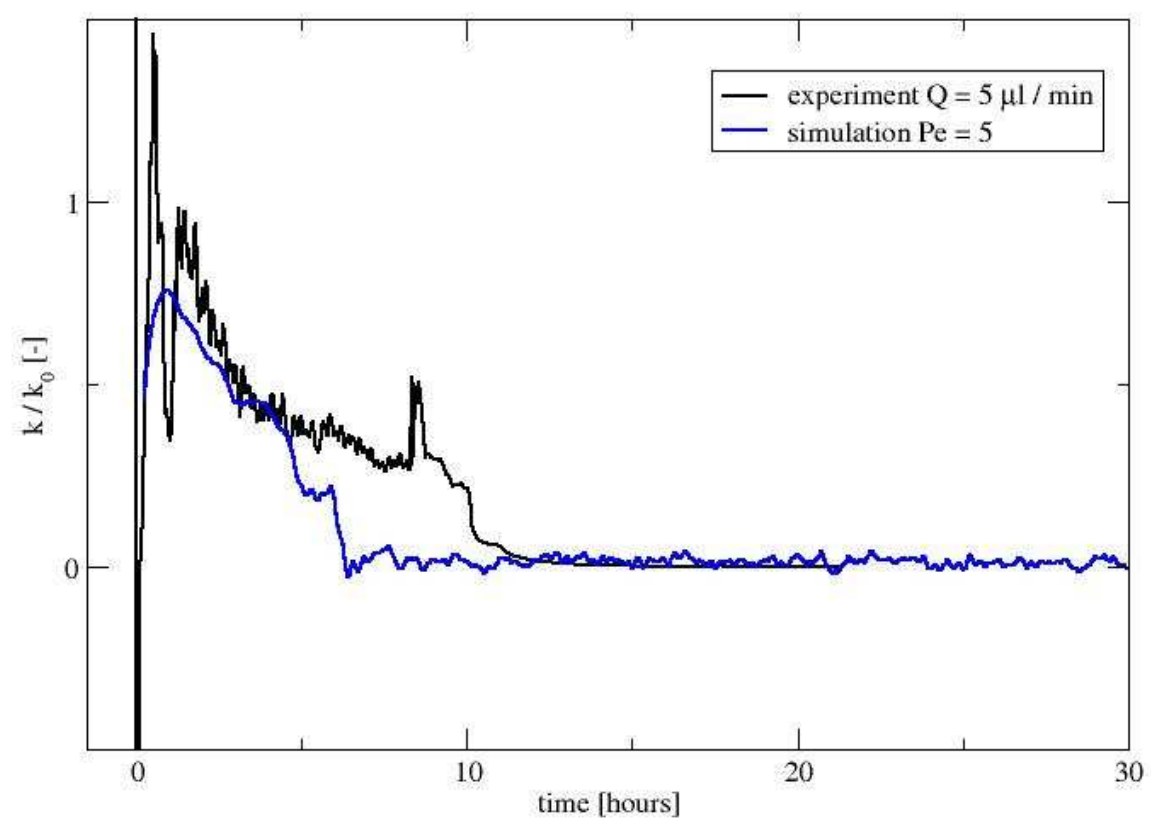

Figure 12. Dimensionless conductivity $k / k_{0}$ of the capillary as a function of time for, for both experiment $(Q=5 \mu \mathrm{l} / \mathrm{min}$, black curve) and simulation (Pe $=5$, blue curve)

From this figure, we observe that full blocking of the capillary occurs after roughly 7 hours in the simulation and 10 hours in the experiment. Furthermore, we observe that the time evolution of the dimensionless conductivity for early times is similar for both experiment and simulation. Finally, we note that, once deposition occurs, it is permanent.

Second, we consider the experimental results for the double volumetric flow rate $Q=10 \mu \mathrm{l} / \mathrm{min}$. The results are presented in Figure 13. 


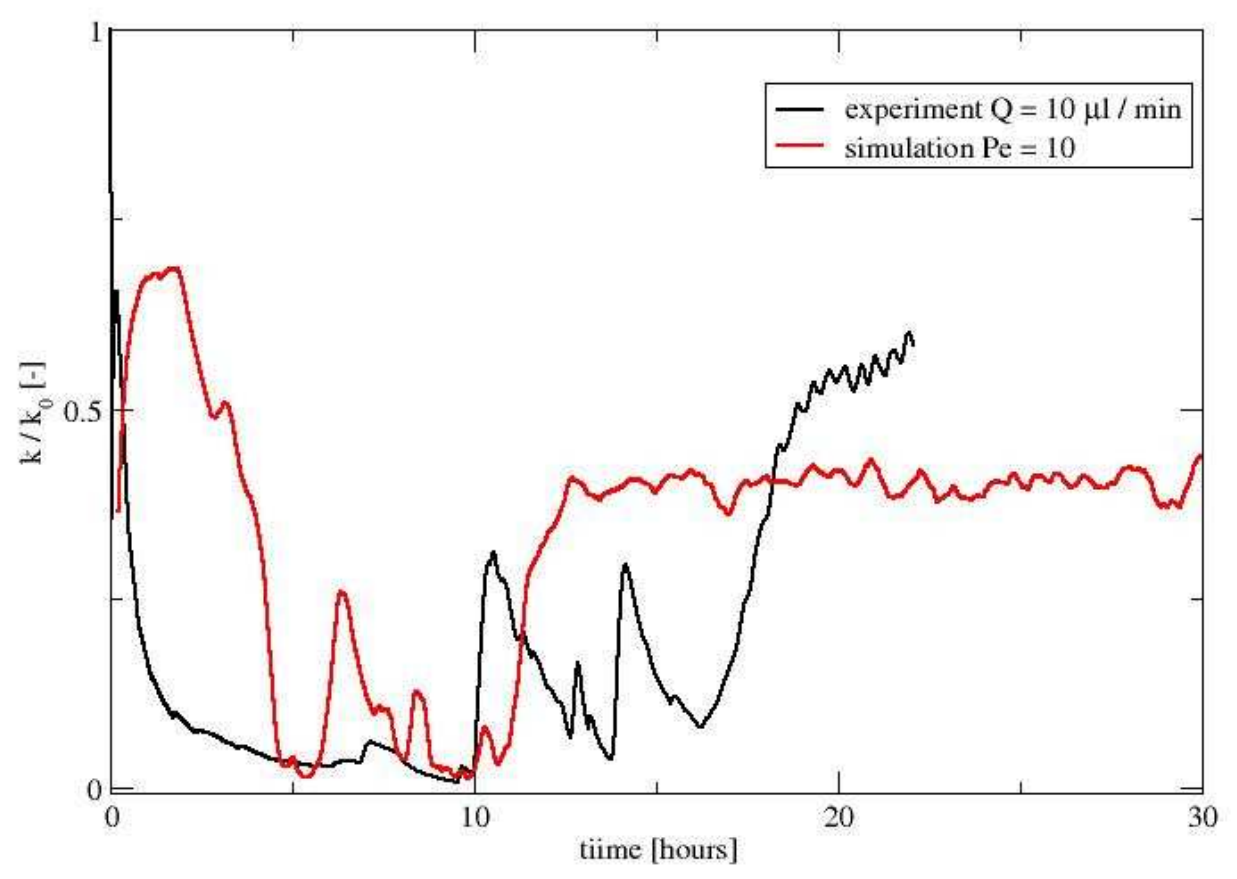

Figure 13. Dimensionless conductivity $k / k_{0}$ of the capillary as a function of time for, for both experiment $(Q=10 \mu \mathrm{l} / \mathrm{min}$, black curve $)$ and simulation $(\mathrm{Pe}=10$, blue curve $)$

We now find that the best comparison with simulation data is for the case $\varepsilon_{\mathrm{cw}}=\varepsilon_{\mathrm{cc}}=10 k_{B} T$ and $P e=$ 10 , that is, at a flow rate doubled compared with the previous simulation case and the same value of $\varepsilon_{\mathrm{cc}}$. From this figure, we observe that initial blocking occurs faster in both the experiment and simulation. In addition, we now observe erosion and redeposition on a time scale of a few hours. Note that the conductivity profiles during these stages show typical saw-tooth behaviour, which is very similar for both experiment and simulation. Third, we observe that the clogging is transient for both experiment and simulation. This is different from the case of $Q=5 \mu \mathrm{l} / \mathrm{min}$, where we observed that the blocking is permanent. Apparently, the shear forces are now strong enough to entrain the asphaltene deposits. 


\section{Discussion and Conclusions}

We have studied the deposition and aggregation of colloidal asphaltene in capillary flow by experiment and computer simulation. The flow experiments were carried out in glass capillaries, where we use extracted asphaltenes in toluene, reprecipitated with $n$-heptane. In the experiments, the dynamics of asphaltene precipitation and deposition were monitored in a slot capillary using optical microscopy in combination with measurement of the pressure drop as a function of time. Maintaining a constant flow rate of $5 \mu \mathrm{L} \mathrm{min}$, we found that the pressure drop across the capillary initially increased slowly. A sharp increase in pressure drop was subsequently observed. Microscopy observations confirm that this corresponds to the increased deposition of asphaltenes on the capillary walls. Doubling the flow rate to $10 \mu \mathrm{L} \mathrm{min}{ }^{-1}$, we observe that the initial deposition occurs faster, but the deposits are subsequently entrained by the flow. To confirm the experimental observations, we have carried out particulate computer simulations using the Stochastic Rotation Dynamics (SRD) method. The asphaltene colloids interact through a screened Coulomb potential. We systematically vary the potential well depth $\varepsilon$, and the flow rate so that $P e^{\text {flow }}>>1$ (hydrodynamic interactions dominate) and $R e<<1$ (Stokes flow). In the simulations, we impose a pressure drop over the capillary length, and measure the corresponding solvent flow rate. We observe that the transient solvent flow rate decreases when the asphaltene particles become more "sticky". For a well depth $\varepsilon=2 k_{B} T$, a monolayer deposits of on the capillary wall. Withincreasing well depth, the capillary becomes totally blocked. The clogging is transient for $\varepsilon=5 k_{B} T$, but appears to be permanent for $\varepsilon=10-20 k_{B} T$. By considering diffusion of the colloidal asphaltene particles, we obtain a time scale for the simulation which matches the experiment. The simulations seem to capture the essential features of the deposition process: permanent blocking for small flow rates and transient blocking for larger flow rates, due to erosion / entrainment. We calculate the change in the dimensionless permeability of the capillary as a function of time for both experiment and simulation. For our asphaltenes (Cold Lake), we find a good comparison between experiments and simulations for $\varepsilon_{\mathrm{cc}} \approx 10 \mathrm{k}_{\mathrm{B}} \mathrm{T}$. This is a reasonable value for colloidal particles. ${ }^{15}$ Our results show that the key 
parameter is the colloidal interaction potential, but this parameter is difficult to measure. Recently, a number of AFM studies have been published to shed light on this subject. ${ }^{23},{ }^{24}$ This interaction can be changed by many different external factors, such as co-precipitated elements, the geometry and hardness of the particles, etc. The method used in this paper allows the identification of such an interaction parameter for the asphaltenes extracted by a given method. However, the situation can be quite different in a production well. We believe that the method suggested by the authors can be useful to identify how different factors will affect the colloidal interaction potential of asphaltene particles.

Finally, by matching the experimental and simulation results, we have obtained information about the asphaltene interaction potential and flow conditions associated with the asphaltene deposition process.

\section{Acknowledgments}

JTP acknowledges the Netherlands Organisation for Scientific Research (NWO) for financial support. 


\section{Nomenclature}

- $\quad a=0.5 *$ expt. capillary slot depth

- $b=0.5 *$ expt. capillary slot width

- $\quad D=$ Stokes-Einstein diffusion coefficient

- $g_{S R D}=$ SRD body force

- $\mathrm{k}_{\mathrm{B}}=$ Boltzmann constant

- $\quad \mathrm{MD}=$ Molecular Dynamics

- $N_{f}=$ number of solvent SRD particles

- $P e^{\text {flow }}=$ Peclet number for flow

- $Q=$ expt. flow rate

- $R e^{p}=$ Reynolds number for the particles

- $\boldsymbol{r}_{i}(t)=$ position of SRD solvent particles

- $\mathrm{SRD}=$ Stochastic Rotation Dynamics

- $\mathrm{T}=$ temperature $[\mathrm{K}]$

- $\boldsymbol{v}_{i}(t)=$ velocity of SRD solvent particles

- $\quad v^{\text {flow }}=$ solvent velocity

- $\mathrm{VACF}=$ velocity autocorrelation function

- $\varepsilon_{\mathrm{cc}}=$ asphaltene - asphaltene interaction potential well depth

- $\phi_{\mathrm{cc}}(\mathrm{r})=$ asphaltene - asphaltene interaction potential as function of distance

- $\varepsilon_{1}, \varepsilon_{2}=$ Yukawa potential parameters for attraction and repulsion, respectively

- $\eta=$ solvent viscosity [Pa.s]

- $\gamma_{\mathrm{av}}=$ average shear rate

- $\mathrm{\kappa}^{-1}=$ Debye screening length $[\mathrm{m}]$

- $\sigma=$ colloid diameter

- $\tau_{\mathrm{D}}=$ time required for an asphaltene colloid to diffuse over its own radius 


\section{References}

${ }^{1}$ D. Broseta, M. Robin, T. Savvidis, C. Féjean, M. Durandeau and H. Zhou, "Detection of Asphaltene Deposition by Capillary Flow Measurements”, SPE 59294 (2000)

2 J. Wang, J. S. Buckley, and J. L. Creek, “Asphaltene Deposition on Metallic Surfaces”, J. Dispersion Sci. and Techn. 25, 287-298, (2004)

${ }^{3}$ A.Hammami and J.Ratulowski, " Precipitation and Deposition of Asphaltenes in Production Systems: A Flow Assurance Overview”, in “Asphaltenes, Heavy Oils, and Petroleomics”, O.C. Mullins, E.Y. Sheu, A. Hammami and A. G. Marshall, Eds., Springer New York, 2007.

${ }^{4}$ E. Ramirez-Jaramillo C. Lira-Galeana and O. Manero, Energy \& Fuels 2006, 20, 1184-1196

${ }^{5}$ Monteagudo J.E.P.; Rajagopal K.; Lage P.L.C., Chemical Engineering Science 2002, 57, 323-337.

${ }^{6}$ Wang, S. and Civan, F., J. Ener. Resour. Tech. 2005, 127, 310

${ }^{7}$ N.H.G. Rahmani, J.H. Masliyah and T.Dabros, (2003). AIChE Journal 2003, 49, 1645-1655.

${ }^{8}$ A. Malevanets and R. Kapral, J. Chem. Phys. 1999, 110, 8605; ibid. 2000, 112, 7260

${ }^{9}$ T. Ihle and D.M. Kroll, Phys. Rev. E 2001, 63, 020201; ibid. 2003, 67, 066705 ; 066706.

${ }^{10}$ J.T. Padding and A.A. Louis, Phys.Rev.E 2006, 74, 031402.

${ }^{11}$ S. Succi, The LB Equation for Fluid Dynamics and Beyond, Oxford University Press, 2001.

${ }^{12}$ M. Venturoli and E.S. Boek, Physica A 2006, 362, 23-29.

${ }^{13}$ N. Kikuchi, C. M. Pooley, J. F. Ryder, and J. M. Yeomans, J. Chem. Phys. 2004, 119, 6388.

${ }^{14}$ J.T.Padding and A.A.Louis, Phys.Rev.Lett. 2004, 93, 220601. 
${ }^{15}$ Israelachvili, J.N, Intermolecular and Surface Forces, 2nd Ed., Academic Press, San Diego, 1991.

${ }^{16}$ H.N.W. Lekkerkerker, W.C.K. Poon, P.N. Pusey, A. Stroobants and P.B. Warren, Europhys. Lett. 20 (1992) 559, 1993; H.N.W. Lekkerkerker et al., Phys. Rev. Lett. 73 (1994) 752; Physical Review E 51 (1995) 558.

17 H. Guerin, J. Phys.: Condens. Matter 10, L527-L532.

18 “Asphaltenes, Heavy Oils, and Petroleomics”, O.C. Mullins, E.Y. Sheu, A. Hammami and A. G. Marshall, Eds., Springer New York, 2007.

19 E.S.Boek, T.Headen and D. Yakovlev, manuscript in preparation (2008)

20 Yudin, I. K.; Nikolaenko, G. L.; Gorodetskii, E. E.; Melikyan, V. R.; Markhashov, E. L.; Frot, D.; Briolant, Y. Journal of Petroleum Science and Engineering 1998, 20, 297-301.

${ }^{21}$ M.P.Allen and D.J.Tildesley, Computer Simulation of Liquids, Oxford University Press, 1990.

${ }^{22}$ B.K. Cook, D.F. Boutt, O.E. Strack, J.R. Williams, S.M. Johnson, “DEM-fluid model development for near-wellbore mechanics", submitted for publication (2007).

${ }^{23}$ Jun Long, Zhenghe Xu, and Jacob H. Masliyah, Langmuir 2007, 23, 6182-6190.

${ }^{24}$ Jun Long, Liyan Zhang, Zhenghe Xu, and Jacob H. Masliyah, Langmuir 2006, 22, 8831-8839. 